\title{
Lusotopie
}

Recherches politiques internationales sur les espaces

issus de l'histoire et de la colonisation portugaises

XVII(2) | 2018

Lusotopie. Concepts et pratiques

\section{Une communauté lusophone avant la lettre ? Les hommes de lettres portugais et brésiliens dans la seconde moitié du XIX ${ }^{\mathrm{e}}$ siècle}

Uma comunidade lusófona "avant la lettre"? Homens de letras portugueses e brasileiros na segunda metade do século XIX

An "Avant la lettre" Lusophone Community? Portuguese \& Brazilian Men of

Letters in the Second Half of the $19^{\text {th }}$ Century

\section{Sébastien Rozeaux}

\section{(2) OpenEdition}

1 Journals

Édition électronique

URL : https://journals.openedition.org/lusotopie/3325

ISSN : 1768-3084

Éditeur

Idemec - UMR 7307

Édition imprimée

Date de publication : 1 décembre 2018

Pagination : 301-328

ISSN : 1257-0273

Référence électronique

Sébastien Rozeaux, "Une communauté lusophone avant la lettre ? Les hommes de lettres portugais et brésiliens dans la seconde moitié du xIx ${ }^{\mathrm{e}}$ siècle », Lusotopie [En ligne], XVII(2) | 2018, mis en ligne le 01 juillet 2021, consulté le 07 décembre 2021. URL : http://journals.openedition.org/lusotopie/3325

Ce document a été généré automatiquement le 7 décembre 2021

Lusotopie 


\title{
Une communauté lusophone avant la lettre ? Les hommes de lettres portugais et brésiliens dans la seconde moitié du XIX ${ }^{\mathrm{e}}$ siècle
}

\author{
Uma comunidade lusófona "avant la lettre"? Homens de letras portugueses e \\ brasileiros na segunda metade do século XIX \\ An "Avant la lettre" Lusophone Community? Portuguese \& Brazilian Men of \\ Letters in the Second Half of the $19^{\text {th }}$ Century
}

\section{Sébastien Rozeaux}

1 L'indépendance politique du Brésil en 1822 est un point de rupture majeur dans la longue histoire impériale du Portugal, puisque l'Amérique portugaise était devenue le "joyau" de cet Empire dès le XVII ${ }^{\mathrm{e}}$ siècle. La rupture est douloureuse pour le Portugal qui ne reconnaît l'indépendance de son ancienne colonie qu'en 1825, sous la pression des Britanniques. S'ensuivent deux décennies de relations tendues, parfois violentes entre les deux pays, lorsque les réactions lusophobes se multiplient au Brésil (Mendes 2010), mais qui n'effacent pas d'un trait de plume la tradition pluriséculaire de relations commerciales, économiques et culturelles dont le périmètre et le cadre se trouvent redéfinis depuis la suppression de l'exclusif colonial en 1808 et l'indépendance en 1822.

$2 \mathrm{Au} \mathrm{XIX}{ }^{\mathrm{e}}$ siècle, alors que l'empire colonial portugais d'Afrique est encore à l'état de chimère, le Brésil et le Portugal métropolitain sont les deux entités politiques majeures de la lusotopie, entendue ici comme ces espaces hérités de l'histoire et de la colonisation portugaise. Or, le Brésil nourrit depuis 1822 des relations complexes, ambiguës avec son ancienne métropole. Certes, la dynastie des Bragance a manœuvré pour garder la main sur l'Amérique portugaise, lors de la proclamation de l'Empire en 1822, mais au prix d'une rupture de ban avec la branche portugaise, lorsque dom Pedro I doit quitter précipitamment le Brésil en 1831 pour regagner le Portugal, laissant la 
place libre à son jeune fils, le prince héritier, sacré empereur dès 1840 , sous le nom de dom Pedro II, après une décennie de troubles, pendant la Régence.

\section{Reconstruire une relation sur les ruines de l'Empire portugais}

3 C'est dans ce contexte de rupture et de reconfigurations post-coloniales ${ }^{1}$ qu'apparait une volonté commune de définir la relation luso-brésilienne à nouveau frais. $\mathrm{Si}$, au sein des élites, nombreux sont ceux qui en Amérique tournent le dos à l'ancienne puissance coloniale, accusée de tous les maux hérités des temps passés, à commencer par l'esclavage, d'autres mobilisent l'idée d'une communauté de destin, d'une fraternité dont l'essor des relations et échanges culturels serait la manifestation la plus tangible, au mitan du siècle. Cependant que le mouvement romantique tend à exalter les spécificités et les identités nationales de chacun des deux pays, des hommes de lettres théorisent l'idée d'une relation privilégiée entre deux nations unies par une histoire, une langue, une religion voire une "race" partagées. C'est pourquoi cet article a pour ambition de retracer la généalogie et les ressorts de ce premier avatar d'une communauté lusophone avant la lettre, bien antérieure à son institutionnalisation inter-étatique sous l'égide du politique, suite à la création de la Communauté des pays de langue portugaise à la fin du $\mathrm{xx}^{\mathrm{e}}$ siècle. Si le terme de "lusophonie" est à l'évidence anachronique, les discours qui esquissent les contours de cet espace informel de partage justifient d'entreprendre cette archéologie de la lusophonie, dans la mesure où celle-ci permet de réfléchir de façon plus large à la nature et aux enjeux propres aux relations culturelles luso-brésiliennes après l'indépendance de 1822. Cette démarche nous semble d'autant plus légitime qu'elle permet en creux de cerner les problèmes et les limites de la construction récente de la lusophonie officielle, compte tenu du poids très contrasté de ses membres et du caractère très fragile d'une institution politique non dénuée d'arrière-pensées post-impériales (Cahen 1997).

En effet, c'est d'abord au Portugal et au sein de la colonie portugaise du Brésil que s'enracinent les discours de l'objectivisation d'une communauté lusophone avant la lettre, dont les hommes de lettres seraient l'incarnation, autrement dit d'une relation privilégiée entre deux nations unies par une communauté de destin, de culture et de langue qui, après les années critiques de l'indépendance, ont vocation à cultiver des échanges féconds et fraternels afin de mieux défendre leurs intérêts réciproques et s'affirmer dans l'espace littéraire international qui se structure dès l'époque moderne depuis son épicentre, Paris (Casanova 2008). Étudier ces relations littéraires entre le Portugal et le Brésil s'inscrit dans une tradition historiographique ancienne, parfois habillée des oripeaux post-impériaux d'une certaine idée de la place du Portugal dans l'histoire mondiale - une posture que l'on retrouve au xIx ${ }^{e}$ siècle aussi, et qui mérite d'être ici historicisée et critiquée.

\section{Le Dicionário bibliográphico português (1858-1870), un marqueur identitaire en gestation}

5 L'idée d'une communauté partagée entre hommes de lettres portugais et brésiliens s'affirme dans la sphère publique au milieu $d u x x^{e}$ siècle, lorsque retombent les 
tensions héritées des événements de 1822 et que le courant migratoire du Portugal vers le Brésil gagne une ampleur inédite : la stabilisation du cadre politique monarchique dans ces deux pays, la normalisation de leurs relations politiques et commerciales, comme l'essor remarqué des Letras Pátrias au Brésil², dans un contexte plus général d'accélération et d'intensification des échanges transatlantiques, constituent des déterminants favorables à la structuration d'une relation privilégiée entre les deux pays.

6 La publication à Lisbonne du Dicionário bibliográfico português, dont les neuf premiers volumes sont rédigés par Inocêncio Francisco da Silva (18101876) et publiés entre 1858 et 1870 , est un repère important pour qui veut retracer la chronologie de ces relations littéraires et, en creux, de cette communauté littéraire lusophone en gestation (Silva 1858-1870). Ici, le qualificatif de "portugais» prend une ampleur qui dépasse de beaucoup le périmètre du seul Portugal, puisque l'ouvrage bio-bibliographique prétend recenser toutes les publications en langue portugaise :

...dans cet espèce de monument levé à la LANGUE PORTUGAISE, qui ne peut qu'être bien reçu par tous ceux qui la parlent et la cultivent dans les diverses régions $\mathrm{du}$ globe, ce serait une omission impardonnable de ne pas inclure les nombreuses œuvres récemment imprimées dans l'empire du Brésil, c'est-à-dire, depuis que son indépendance politique a été proclamée et reconnue.

Silva 1858 , vol. 1 : xxi-xxii

7 La langue portugaise dans son universalité prend ici corps dans cette lusotopie atlantique qui réunit le Portugal et le Brésil. Or, l'entreprise s'avère en 1858 difficile, tant les premières œuvres produites par les Letras Pátrias depuis la fin des années 1830 au Brésil sont encore méconnues au Portugal. Ainsi, seuls une dizaine d'auteurs brésiliens sont l'objet d'une notice dans ce premier volume. L'idée d'une communauté de destin entre les milieux littéraires portugais et brésilien est encore balbutiante et utopique en 1858, tant l'ignorance (voire l'indifférence) est grande de part et d'autre de l'Atlantique.

Pourtant, la parution volume après volume de la première série de ce dictionnaire permet de suivre pas à pas l'intensification remarquable de ces circulations et la définition des premiers contours d'une communauté d'esprit et d'intérêts. Le séjour de nombreux hommes de lettres brésiliens au Portugal, l'essor d'une colonie portugaise à Rio de Janeiro et la structuration d'un marché éditorial transatlantique pour alimenter les librairies brésiliennes ont permis de mieux informer le bibliographe portugais, dont l'œuvre témoigne de la progressive structuration d'un espace littéraire luso-brésilien non exclusif - tant la relation avec la France est pour ces deux pays essentielle - mais néanmoins bien réel. Inocêncio Francisco da Silva a ainsi bénéficié à partir de 1860 de l'aide d'intercesseurs précieux au sein de la colonie portugaise de Rio de Janeiro: les frères Mello Guimarães, nés à Aveiro et résidents à Rio de Janeiro depuis 1845, travaillent dans le commerce et la banque. Leur parcours s'inscrit dans les voies de cette émigration du Nord du Portugal vers le Brésil, dont l'essor date précisément des années 1840-1850. Dès lors, Inocêncio Francisco da Silva, bibliophile érudit et casanier, acquiert une renommée certaine dans la capitale brésilienne, où son œuvre est reçue avec curiosité puisqu'il contribue à la fois à créer cette communauté de partage sur un pied d'égalité apparent et à promouvoir et internationaliser une jeune littérature brésilienne en mal de consécration et de publicité hors des frontières de l'Empire. Cette initiative privée reçoit ainsi dès 1859 l'appui de la principale institution culturelle de l'Empire, l'Instituto Histórico e Geográfico Brasileiro, dont les réunions se tiennent sous la 
protection de l'empereur. Joaquim Caetano Fernandes Pinheiro loue en ces termes les mérites du dictionnaire portugais :

En incluant dans son périmètre les auteurs brésiliens, l'énumération résumée de leurs écrits et notices biographiques, Inocêncio Francisco da Silva concourt à resserrer les liens entre les deux peuples, qui ont en partage une origine commune, professent une même religion et parlent une même langue, qui se situent aux marges opposées de l'Atlantique et qui conservent leur autonomie respective, et forment une grande et puissante race ${ }^{3}$.

Inocêncio Francisco da Silva obtient le titre de membre correspondant de l'Institut puis, en 1863, dom Pedro II lui confère le titre de chevalier de l'Ordre de la Rose, suite à la publication de la première série complète de son dictionnaire, qui compte alors sept volumes. En annexe à ces volumes se trouvent des extraits de la presse brésilienne et portugaise qui témoignent de l'accueil bienveillant réservé à sa publication, de part et d'autre de l'Atlantique. Dans la célèbre Revista Popular publié à Rio de Janeiro par Baptiste-Louis Garnier, Joaquim Norberto de Sousa Silva salue les mérites d'une œuvre qui exalte "l'esprit luso-brésilien" ${ }^{4}$. Fort de cette réputation nouvelle, le bibliographe est sollicité par des écrivains et éditeurs brésiliens afin qu'il inclue leurs œuvres les plus récentes dans le dictionnaire. De très nombreuses notices attestent de cette correspondance transatlantique dont Inocêncio Francisco da Silva est le récipiendaire. À tel point qu'il devient l'un des meilleurs connaisseurs des Letras Pátrias du Brésil au Portugal, comme en témoignent les nombreuses biographies d'écrivains brésiliens qu'il rédige pour le compte de la presse portugaise. $\mathrm{Vu}$ du Brésil, ce dictionnaire est perçu par les éditeurs comme une possibilité rare et donc précieuse de promouvoir leur catalogue, et donc les Letras Pátrias, en Europe, via le relais du Portugal. En retour, les frères Laemmert, éditeurs à Rio de Janeiro, proposent à la vente le dictionnaire et louent dans leur catalogue une œuvre qui exalte "les richesses littéraires des deux pays frères" (Dicionário 1867). L'éloge de cette confraternité littéraire se double d'ambitions éditoriales et économiques bien comprises, avec l'espoir de voir se développer un marché transatlantique du livre qui puissent bénéficier tant aux éditeurs portugais qu’à ceux du Brésil.

Toutefois, la réception au Brésil de ce dictionnaire est loin d'être univoque: les postulats de confraternité et de parenté entre les deux nations achoppent à convaincre tout à fait, lorsque l'on s'arrête sur les décalages manifestes dans sa réception de part et d'autre de l'Océan Atlantique. Ainsi, les articles parus dans la presse portugaise ne font que rarement mention de la dimension «brésilienne » de cet ouvrage, et voient plutôt dans celui-ci la marque de la renaissance d'une nation affaiblie depuis 1808. Au Brésil, des voix se font entendre pour pointer les défauts d'un dictionnaire qui tend à faire la part belle au Portugal. Dans Le Brésil littéraire (1863), Ferdinand Wolf en appelle à la méfiance vis-à-vis du Portugal, lorsqu'il évoque le dictionnaire d'Inocêncio Francisco da Silva: "Les sentiments de jalousie qu'on a pour le Brésil, l'air de dédain avec lequel on considère cette ancienne colonie, ne permettent pas d'accorder à sa littérature une position indépendante à côté de celle du Portugal" (Wolf 1863). En somme, l'historien de la littérature brésilienne dévoile les ressorts cachés de l'impérialisme portugais sous couvert d'une supposée confraternité littéraire luso-brésilienne.

11 De telles réserves ne sont pas sans lien avec le lancement en 1883 du Dicionário bibliográfico brasileiro (Blake 1883-1902). Sacramento Blake mentionne très brièvement dans l'introduction "l'important dictionnaire d'Inocêncio", des mots choisis pour qualifier ce précédent incontournable dont il préfère souligner les nombreuses lacunes. 
Dans ce nouvel opus bibliographique, la place faite aux lettres portugaises est nulle. L'ambiguïté originelle de cette communauté luso-brésilienne louée par certains, dénoncée par d'autres, s'expose ici dans la concomitance entre la parution du premier volume du dictionnaire bibliographique brésilien et celle d'une nouvelle série du Dicionário bibliográphico português, à l'initiative de l'homme de lettres portugais Brito Aranha, qui souligne en introduction combien son initiative a été reçue positivement au sein de la "république littéraire» au Brésil, puisqu'il dit avoir réceptionné des centaines d'ouvrages et de notices fournies par des éditeurs et écrivains brésiliens soucieux de figurer dans le dictionnaire portugais. Ainsi, l'idée d'une communauté partagée au sein d'une république des lettres de langue portugaise s'affirme dans la sphère publique sans pour autant faire consensus au Brésil ${ }^{5}$.

\section{Fraternité ou paternalisme?}

En 1879, Camilo Castelo Branco fait paraitre une anthologie de poésie joviale et humoristique, le Cancioneiro alegre de poetas portuguezes e brazileiros, dans laquelle il reprend à son compte l'idée d'une communauté lusophone avant la lettre, dont certaines compositions se font l'écho. Camilo Castelo Branco (1825-1890), l'une des principales personnalités du monde littéraire portugais, est alors au faîte de sa carrière. Homme de lettres polygraphe et auteur prolifique, il témoigne dès les années 1850 d'un intérêt marqué pour le Brésil, à la fois source d'inspiration, de curiosité voire de revenus, lorsqu'il apparaît que le marché du livre au Brésil peut offrir des perspectives intéressantes aux auteurs portugais ${ }^{6}$. Parmi la soixantaine de poètes dont l'œuvre est citée et commentée figure une demi-douzaine de poètes brésiliens. Trois de ces poètes sont originaires de la province du Maranhão, dont la capitale São Luís se vante d'être au XIX ${ }^{e}$ siècle une "nouvelle Athènes" qui cultive des relations privilégiées avec l'ancienne métropole portugaise (Rozeaux 2014b). Plus largement, le recueil fait la part belle à ces lettrés qui incarneraient de façon exemplaire la fécondité des échanges entre les deux nations: Camilo ne manque pas de rappeler que Gonçalves Dias comme Casimiro de Abreu ont entamé leur carrière littéraire au Portugal. Il accorde aussi dans son recueil une place de choix à Faustino Xavier de Novais (1820-1869), un homme de lettres qui a quitté le Portugal pour s'installer à Rio de Janeiro, ou au poète Gonçalves Crespo (1846-1883), né à Rio de Janeiro, qui vit au Portugal depuis l'âge de dix ans.

13 Cette compilation est agrémentée de commentaires écrits dans un style volontiers ironique et polémique qui suscite des réactions outragées au Portugal, lorsque Camilo Castelo Branco s'en prend à la jeune génération des poètes "modernes", comme au Brésil, à tel point que paraît quelques mois plus tard un opuscule d'une soixantaine de pages intitulé Os criticos do Cancioneiro alegre (Branco 1879). La date de parution témoigne d'une circulation rapide et d'échanges soutenus au sein de l'espace littéraire transatlantique, puisque cet opuscule compile les recensions critiques "bienveillantes", exclusivement portugaises, et les réponses très sarcastiques que Camilo adresse à ses détracteurs, qu'ils soient portugais ou brésiliens. À Rio de Janeiro, la Revista illustrada évoque les réactions en chaîne provoquées par le Cancioneiro alegre à l'été 1879 :

La littérature s'est fait remarquer cette semaine par le petit bruit qu'a produit ici le Cancioneiro alegre, boulet que Camilo Castelo Branco nous a lancé depuis les contrées lusitaniennes et qui a mis en effervescence toute la jeunesse de cette terre. Des réponses, des lettres, des articles, un déluge de mots destinés à Camilo qui n'en attend pas moins, il me semble ${ }^{7}$. 
14 reproche d'abord au Portugais d'avoir choisi les œuvres les moins célèbres des poètes brésiliens afin de mieux prêter le flanc à la satire. Certains mettent en cause la mauvaise foi du compilateur, trop prompt à moquer la verdeur des talents littéraires du Brésil, quand d'autres y voient surtout le reflet d'une méconnaissance coupable des richesses des Letras Pátrias. Dans tous les cas, la déception est grande : là où le lecteur brésilien semblait espérer que le compilateur traite avec "fraternité" les poètes inclus dans son recueil, les commentaires de Camilo usent plutôt de l'ironie pour moquer les travers des auteurs brésiliens en des termes qui renvoient à leurs prétendues origines indigènes voire animales. D'où la virulence des critiques brésiliennes et des répliques de Camilo dans l'opuscule de 1879 où, loin de vouloir apaiser la situation, il affirme dans ce style péremptoire si particulier: "Pedro Alvares Cabral a découvert le Brésil ; moi je suis en train de découvrir les idiots qui le peuplent". qu'elle met en évidence de part et d'autre de l'océan Atlantique. La crispation presque unanime des critiques brésiliens témoigne d'un sentiment national exacerbé, caractéristique de cette époque de formation des Letras Pátrias, soucieuses de cultiver leur indépendance vis-à-vis de l'ancienne métropole. À propos du Cancioneiro alegre, Isaias d'Almeida explique « la très profonde ignorance » de la littérature brésilienne au Portugal par le fait que « les écrivains portugais s'entêtent avec opiniâtreté à nier » son existence (Almeida 1879). Plus sévère encore, le célèbre critique et historien des lettres Sílvio Romero tire à la fin de l'année 1879 les leçons de cette polémique dans un article paru dans la Revista brazileira. À l'y croire, les attaques subies seraient la conséquence pathétique d'un basculement des forces au profit du Brésil :

Le vieux royaume a définitivement perdu son charme à nos yeux. D'où une certaine exaspération qui s'empare de temps à autre des écrivains portugais lorsqu'ils traitent du Brésil ou des Brésiliens. Les exemples des Farpas ${ }^{9}$ et du Cancioneiro alegre sont encore très récents. Il s'agit là d'un symptôme pathologique évident de l'apathie intellectuelle du vieux royaume ${ }^{10}$.

À travers la personnalité éminente de Camilo, dont la cible semble idéale puisqu'il est alors considéré comme le parangon des lettres portugaises, il s'agit pour ses détracteurs brésiliens, plus ou moins virulents, de dénoncer les postures paternalistes qui se cachent trop souvent derrière le paravent de la fraternité. Si la connaissance réciproque des littératures nationales, contemporaine de l'essor des circulations littéraires transatlantiques, est une réalité tangible à partir des années 1860-1870, elle s'accompagne donc de tensions et de rivalités continues. Ainsi, cette polémique de quelques mois témoigne des profonds malentendus et clivages qui empêchent la constitution d'un espace littéraire luso-brésilien apaisé, comme elle reflète pourtant la réalité des circulations littéraires sans lesquelles celle-ci n'aurait pas même existé : la réplique immédiate et multiple, de part et d'autre, atteste l'intensité de ces échanges et l'intérêt qui entoure la réception des Letras Pátrias au Portugal, à laquelle les écrivains brésiliens sont tout sauf indifférents ${ }^{11}$. 


\section{4 “Le Brésil, terre d'avenir" des hommes de lettres portugais}

17 Certes, rares sont au XIX ${ }^{e}$ siècle les écrivains portugais qui quittent leur pays pour faire carrière au Brésil. On peut citer les noms du poète Faustino Xavier de Novais, beaufrère de Machado de Assis, d'Emílio Augusto Zaluar, de José Feliciano de Castilho ou encore de l'acteur et impresario Furtado Coelho, grande figure de la scène dramatique brésilienne. Pourtant, il semble que le Brésil a été perçu par la grande majorité des écrivains portugais au xIX siècle comme une "terre d'avenir", comme un horizon vers lequel se tourner afin de faire meilleure carrière. Leurs discours révèlent en creux les contours d'une lusophonie avant la lettre, comme légitimation d'une connexion étroite entre le Portugal et le Brésil.

18 La perte du joyau de l'Empire en 1822, un empire africain encore tissé de mots, la transition libérale et la guerre civile qui s'en suit dans les années 1840, l'endettement structurel du pays, sa sujétion politique, militaire et diplomatique à l'encombrant allié britannique, la crise économique et l'essor exponentiel de l'émigration vers le Brésil, sont autant d'éléments qui alimentent un discours de la décadence nationale au sein des élites du royaume. Et la littérature ne fait pas exception : beaucoup d'hommes de lettres dénoncent la modestie de la création littéraire, le manque de lecteurs et la précarité de leur situation professionnelle, à l'instar de Pinheiro Chagas en 1879 :

Si la littérature portugaise est stérile, quelle en est la raison ? C'est simplement parce que la culture des lettres ne rémunère pas suffisamment qui s'y consacre, et les nécessités incontournables de la vie l'obligent fatalement à suivre une autre voie. Le Brésil nous offre un marché vaste et accueillant...

Chagas $1879: 69$

19 Ces considérations concluent une longue lettre publique adressée à l'empereur Dom Pedro II dans laquelle le dramaturge portugais défend la propriété littéraire et la nécessité de conclure une convention internationale entre les deux pays afin de protéger les intérêts financiers des écrivains portugais. Cette lettre publiée à Lisbonne et reproduite dans la presse brésilienne résume parfaitement les aspirations nouvelles des écrivains portugais vis-à-vis du marché brésilien: tout comme les émigrés portugais qui gagnent le Brésil pour faire fortune ou à tout le moins améliorer leurs conditions de vie, les écrivains portugais ont tôt fait de se tourner vers l'ancienne colonie dans l'espoir d'augmenter leurs revenus, cependant que la littérature portugaise connaît une circulation croissante dans l'Empire, par le biais de l'importation, mais aussi du plagiat ou de la contrefaçon, alors monnaie courante.

20 Au Portugal, les premières plaintes contre la contrefaçon au Brésil datent des années 1840, lorsqu'Alexandre Herculano dénonce en 1843 dans la revue Panorama la "piraterie" des journaux brésiliens qui pillent certains titres de la presse portugaise pour remplir leurs colonnes. C'est en 1851 qu'une autre figure tutélaire du romantisme portugais, Almeida Garrett, rédige et fait adopter une loi sur la propriété littéraire au Portugal. Nommé ministère des Affaires Étrangères en 1852, il missionne le consul du Portugal à Rio de Janeiro pour entamer des discussions en vue de la signature d'une convention littéraire luso-brésilienne, afin de défendre les intérêts des éditeurs et écrivains portugais au Brésil, et réciproquement. Le conseil des ministres de l'Empire pointe le caractère déséquilibré du marché luso-brésilien du livre et de l'imprimé pour rejeter une telle idée jugée peu bénéfique pour le Brésil. Parmi les contempteurs d'une 
telle initiative, l'empereur joue un rôle primordial, soucieux qu'il est de défendre une conception classique de la création littéraire, qui use des sinécures et du mécénat impérial afin d'assurer de bonnes conditions de vie aux écrivains de cour (Rozeaux 2014a).

Ces blocages de la part de l'Empire brésilien accroissent l'exaspération des écrivains portugais qui se sentent floués, car leurs œuvres sont reproduites et rééditées sans qu'ils ne touchent aucun bénéfice. Comme Pinheiro Chagas, Camilo Castelo Branco dénonce dans la presse le tort que lui causent les contrefacteurs, alors qu'il espère accroître ses revenus par la vente de ses droits au Brésil, où ses romans circulent abondamment. Il est soutenu en cela par son éditeur, le Français Ernest Chardron, dont la maison d'édition sise à Porto développe des relations commerciales privilégiées avec le Brésil, comme par la très large majorité des écrivains portugais soucieux, depuis l'adoption de la loi de 1851, d'étendre la jouissance de leurs droits au Brésil. Cette propagande collective des écrivains portugais, avec le relais de la diplomatie portugaise, témoigne à l'évidence des intérêts matériels, économiques qui déterminent de façon sous-jacente l'espace littéraire luso-brésilien.

Or, à compter des années 1860-1870, une alliance se noue entre hommes de lettres portugais et brésiliens sur ce sujet, lorsqu'une majorité de ces derniers se convertit au modèle de l'écrivain professionnel, dont la carrière dépend moins des faveurs clientélistes que des revenus tirés de la vente de ses publications. Ainsi, la lettre que Pinheiro Chagas adresse à l'empereur en 1879 circule à Rio de Janeiro, où elle est "assez bien lue et connue" (Coelho 1880: 498). S'il y consacre le principe universel de la propriété littéraire, l'auteur insiste également sur le lien spécifique qui unirait le Brésil au Portugal: "Messieurs, les écrivains ne sont pas la propriété exclusive du pays où ils sont nés. Leur patrie est au moins composée de tous les coins de la terre où l'on parle leur langue" (Chagas 1879: 63). Ainsi, il ne s'agirait pas de défendre les intérêts particuliers des écrivains portugais mais la cause des écrivains de langue portugaise. De fait, cette lettre bénéficie du relais du principal organe de presse brésilien, la Gazeta de Notícias, comme de la presse (plus confidentielle) de la colonie portugaise de Rio de Janeiro. D’ailleurs, le quotidien libéral fondé en 1875 par José Ferreira de Sousa Araújo est le fer de lance de la défense des droits des écrivains. Voilà pourquoi de nombreuses plumes brésiliennes et portugaises associent alors leur nom à un journal réputé offrir les meilleures rémunérations de la presse de langue portugaise - et ce bien avant la signature en 1889 d'une première convention littéraire luso-brésilienne et l'adoption en 1891 du nouveau Code pénal des États-Unis du Brésil, dans lequel l'article 345 reconnaît enfin la propriété littéraire et artistique (Rozeaux 2017).

\section{Les correspondants portugais de la presse brésilienne en Europe, au cœur de l'espace littéraire luso-brésilien}

$\mathrm{Au}$ Brésil, les bons usages en matière de propriété littéraire ont précédé la loi. En particulier, l'installation de la maison Garnier à Rio de Janeiro a permis à de nombreux écrivains de tirer des revenus importants de la cession de leurs droits sur telle ou telle œuvre. En 1869, Pinheiro Chagas s'adresse d'ailleurs à Baptiste-Louis Garnier afin qu'il veille à faire représenter et imprimer ses œuvres dramatiques au Brésil. Il semblerait que l'éditeur n'ait pas donné suite à cette requête. Et le fait même de publier dix années 
plus tard la lettre publique mentionnée ci-dessus témoigne de l'amertume du dramaturge vis-à-vis du traitement qui lui est réservé au Brésil. C'est à un autre éditeur, António Augusto da Cruz Coutinho, originaire de Porto, qu'il revient de vendre les œuvres du dramaturge portugais, comme l'attestent les nombreuses annonces publiées dans la presse carioca. Les registres d'entrée des navires transatlantiques nous apprennent que cet éditeur reçoit régulièrement des caisses de livres en provenance du Portugal pour achalander les rayons de sa Livraria popular, qui ouvre en plein centre de Rio de Janeiro en 1866 :

On trouve dans cette nouvelle librairie toutes les œuvres de Camilo Castelo Branco, Alexandre Herculano, Almeida Garrett, Rebello da Silva, Mendes Leal, Conselheiro Bastos, Zaluar, José de Alencar, Dr. Macedo, Cosino Louzada, Arnaldo Gama, Bulhão Pato, Teófilo Braga, Castilho, Pinheiro Chagas, Paul de Kock, et de nombreux autres écrivains dont les œuvres sont en vente aux prix les plus raisonnables possibles, puisqu'on les reçoit directement de Porto et de Lisbonne ${ }^{12}$.

Voilà un libraire portugais installé à Rio de Janeiro qui offre un large choix de littérature portugaise contemporaine, outre qu'il propose également dans son catalogue les grands noms des Letras Pátrias brésiliennes. Il est un de ces passeurs dont l'activité économique contribue à construire cet espace littéraire luso-brésilien comme espace privilégié, bien que non exclusif, de circulations du livre et de l'imprimé. Dans les années 1880, Cruz Coutinho se spécialise dans la librairie populaire, en proposant des ouvrages portugais et français à un prix très concurrentiel, preuve de la vitalité de ce marché transatlantique du livre qui permet de mieux assoir l'hégémonie des librairies portugaises et françaises au Brésil. Cela signifie que les auteurs portugais vendus à Rio de Janeiro chez ce libraire tiraient quelques revenus de la vente de leurs ouvrages, et ce avant même la signature de la convention littéraire bilatérale de 1889 .

Les bénéfices tirés du marché du livre et de l'imprimé au Brésil sont encore plus nets lorsque l'on se tourne vers la presse quotidienne. Toutes choses égales par ailleurs, c'est une première "civilisation du journal" (Kalifa et al. 2011) qui naît dans les milieux urbains brésiliens au cours des trois dernières décennies du XIX ${ }^{\mathrm{e}}$ siècle, à la faveur de l'émergence d'une nouvelle presse quotidienne plus accessible, à même de satisfaire les attentes de ces classes moyennes alphabétisées qui peuplent les villes, ces archipels lettrés dans le vaste océan de l'analphabétisme, comme le révèlent les données du premier recensement de $1872^{13}$. Cette presse a adopté très tôt le modèle du roman feuilleton et a publié de façon abusive bien des auteurs français et portugais depuis les années 1840. Les choses évoluent à partir des années 1870, quand cette nouvelle presse recourt aux services rémunérés de plumes portugaises pour alimenter la chronique des actualités européennes et les feuilletons (Rozeaux 2015a).

La parution en 1875 de la Gazeta de noticias promeut un nouveau modèle directement inspiré de la presse populaire à bas coût qui se développe en Europe occidentale et aux États-Unis depuis quelques décennies déjà (Leal 2015). José Ferreira de Sousa Araújo fonde un nouveau modèle économique dont la survie repose d'abord sur une hausse $\mathrm{du}$ tirage et des ventes, et de manière plus traditionnelle sur les revenus tirés des " insertions à la demande » et des annonces. C'est aussi le premier quotidien à être vendu au numéro, via un réseau de distribution inédit dans la capitale impériale. À la fin du siècle, le journal tire à 40000 exemplaires, alors que d'autres journaux populaires sont également apparus dans le paysage médiatique carioca, comme A Gazeta da Tarde (1880), o País (1884), A Notícia (1884), o Diário de Notícias (1885), A Cidade do Rio (1888) ou le Jornal do Brasil (1891). 
27 Le succès rencontré par la Gazeta de Notícias tient en partie à ses correspondants de renom en Europe, et en particulier à ces hommes de lettres portugais qui jouissaient déjà d'une grande réputation au Brésil, où leurs œuvres circulent abondamment, essentiellement via la contrefaçon. Il faut ajouter à ce premier portrait de groupe un effet de "génération", compte tenu du fait que la plupart des correspondants de la Gazeta de Notícias ont été membres de la "génération de [18]70". Le premier "Cénacle" qui se réunit depuis 1868 dans la demeure de Jaime Batalha Reis à Lisbonne est le prélude à la tenue des célèbres "Conférences démocratiques du Casino de Lisbonne", entre mai et juin 1871, comme à la fondation du journal A República en 1870. Le recrutement de ces jeunes hommes de lettres portugais n'est pas pour surprendre de la part de Ferreira de Araújo, quand l'on sait ses convictions abolitionnistes, républicaines et progressistes. Sur le plan littéraire, la Génération de 70 a incarné une profonde rénovation des normes et des pratiques littéraires. Or, compte tenu de l'intense circulation des idées, des hommes et des imprimés en provenance du Portugal, cette nouvelle génération acquiert une solide réputation au Brésil (Berrini 2003). D’autant plus que ces hommes de lettres étaient engagés dans des trajectoires de carrière où l'horizon atlantique est perçu comme une opportunité d'accroître leur réputation et leurs revenus : les collaborations à la presse brésilienne sont en effet mieux rétribuées et cette publicité offerte par la presse brésilienne leur permet d'espérer de meilleures ventes de leurs ouvrages au Brésil. Pour preuve, cette confession d'Eça de Queirós à son ami Oliveira Martins, dans une lettre datée du 23 mai 1888, afin de justifier sa décision de publier de façon simultanée son dernier roman, La Correspondance de Fradique Mendes, dans les journaux 0 Repórter de Lisbonne et la Gazeta de Notícias de Rio de Janeiro. Il s'agit pour lui ici de compenser le salaire dérisoire que lui verse le journal portugais pour ses feuilletons, sachant que "dans les journaux du Brésil ils [lui] rapporteraient le double" (Berrini 2003 : 120).

Les noms des principaux collaborateurs sont mis en avant lors des campagnes de réabonnement lancées par le journal à la fin de chaque année. Ce sont là des éléments de distinction dont la plupart des titres de la presse carioca se prévalent pour séduire le lecteur. Parmi les membres de cette «Génération de 70 » figure l'un des collaborateurs les plus assidus de la Gazeta de Notícias, Ramalho Ortigão (1836-1915), qui est l'auteur de plus de 500 articles parus dans ce journal entre 1879 et 1915 (Zam 2009). Son recrutement fait suite au succès retentissant et polémique que suscite la publication des Farpas dans les années 1870. Cette œuvre écrite à ses débuts en collaboration avec Eça de Queirós constitue une illustration paradigmatique de l'ambigüité de cette communauté lusophone avant la lettre dont l'essor s'accompagne de crispations nationalistes. En juillet 1872, Ramalho Ortigão a eu ces mots catégoriques : “Telle est la vérité, tous les Portugais qui sont allés au Brésil le disent, comme tous les Brésiliens doués d'intelligence : le Brésilien déteste le Portugais"14. Quelques mois plus tard, dans une livraison consacrée à l'émigration portugaise au Brésil, il dresse un portrait au noir de l'Empire des Bragance, notamment sur le plan culturel: "On ne publie pas un seul livre didactique, expérimental ou scientifique. Tout juste si quelques poètes moribonds, languides, voluptueux, inspirés, soupirent dans les hamacs suspendus au tronc des palmiers"15. Pourtant, en 1887, à l'invitation de Ferreira Araújo, ce même Ramalho Ortigão séjourne pour la première fois au Brésil où il est reçu en grande pompe. Une brève parue dans Cidade do Rio nous éclaire sur les mondanités luso-brésiliennes qui ponctuent le passage du célèbre homme de lettres portugais dans la capitale: "En hommage au grand écrivain, qui fait l'honneur et la fierté de la langue et de l'esprit 
portugais de part et d'autre de l'Atlantique, notre illustre collègue Ferreira de Araújo, rédacteur de la Gazeta de Notícias, réunit aujourd'hui les littérateurs et journalistes dans son palais, quai de la Gloria"16.

Ami et collaborateur de Ramalho Ortigão, Eça de Queirós devient en 1877 un correspondant assidu de la Gazeta de Notícias, depuis Londres puis Paris. Le plus célèbre romancier portugais jouit au Brésil d'une solide réputation, comme en témoigne ce commentaire paru dans la Gazeta de Notícias lors de son recrutement: "Inutile de renchérir sur les mérites de notre nouveau collaborateur, dont le nom est associé à ces travaux de grande valeur littéraire. Comme en témoignent les Farpas, $O$ Crime do padre Amaro, $O$ Primo Basilio et quelques autres écrits d'excellente facture" ${ }^{17}$. La qualité et l'importance de cette collaboration prennent une nouvelle dimension avec la parution du "Supplément littéraire" en 1892 (Bonilha 2015). Pour nourrir les deux pages très denses de ce supplément culturel et scientifique, son directeur Eça de Queirós peut compter sur la collaboration de plusieurs hommes de lettres portugais et brésiliens.

Cette collaboration se poursuivra jusqu'à la mort de ce dernier en $1900^{18}$. À l'annonce de son décès, la Gazeta de Notícias fait d'ailleurs célébrer une messe funèbre en son honneur à Rio de Janeiro et lui rend hommage à la une du journal. Six années plus tôt, le journal avait déjà rendu un vibrant hommage à l'un de ses collaborateurs portugais les plus célèbres, Olivieira Martins (1845-1894). Le journal salue la mémoire de cet intellectuel dont la mort est "une énorme perte pour tous les peuples qui parlent la langue portugaise"19. Et le journaliste de poursuivre: "Au Brésil, aucun écrivain portugais n'était plus lu, admiré et discuté que lui."

\section{La presse luso-brésilienne, une utopie lusophone aux racines portugaises}

Il est aussi une autre catégorie de la presse qui a ouvert de nouveaux horizons atlantiques aux hommes de lettres portugais. Je veux parler de la presse lusobrésilienne, un phénomène éditorial essentiellement portugais, qui fait le pari d'une ouverture au public brésilien afin d'augmenter son lectorat et faire vivre cette communauté d'intérêt et d'esprit entre les deux pays. Si la plupart de ces entreprises éditoriales sont éphémères, certains titres rencontrent un succès plus durable. Parmi ceux-ci figurent A illustração luso-brazileira. Jornal universal (1856-1859), la Revista Contemporânea de Portugal e Brasil (1859-1865), la Revista occidental (1875), Os dois Mundos : illustração para Portugal e Brazil (1877-1881), Occidente. Revista illustrada de Portugal e do estrangeiro (1878-1914), o Atlantico (1880-1897), A Illustração. Revista quinzenal para o Brazil e Portugal, (1884-1892), ou la Revista de estudos livres (1883-1886). Autant de publications lisboètes qui ambitionnent de se projeter dans cet espace littéraire luso-brésilien pour faire vivre cette communauté lusophone dont chaque titre prétend être le relais et le promoteur.

Cette presse luso-brésilienne peut prendre des formes diverses, selon la périodicité, la nature de la publication et la place allouée au Brésil dans ses colonnes. Ainsi, A illustração luso-brazileira, fondée en 1856, est une revue qui compte à ses débuts dix-huit collaborateurs, tous portugais. C'est une revue portugaise, destinée d'abord à un public portugais, dans laquelle le Brésil occupe une portion congrue. De même, le texte inaugural de la Revista Contemporânea de Portugal e Brasil, revue littéraire de très bonne 
facture, ne fait étrangement aucune mention du Brésil, en 1859. La place du Brésil s'étoffe au cours de la deuxième année de publication, avec la parution de plusieurs portraits d'écrivains brésiliens contemporains. En 1861, enfin, la revue inaugure une correspondance depuis Rio de Janeiro, tenue par Faustino Xavier de Novais, outre qu'elle recourt aux services de l'historien maranhense João Francisco Lisboa, alors en séjour à Lisbonne, et d'Inocêncio Francisco da Silva. Ici, la dimension luso-brésilienne s'affirme peu à peu, même si la rédaction, l'édition et la diffusion de la revue restent le fait d'acteurs très majoritairement portugais. Cette revue témoigne de ces échanges et circulations transatlantiques nouvelles à l'orée des années 1860, et de l'intérêt croissant au Portugal pour la vie littéraire du Brésil.

Deux décennies plus tard, Mariano Pina, correspondant portugais de la Gazeta de Notícias, fonde à Paris une revue illustrée promise à un grand avenir, A Illustração (1884-1892). Mariano Pina écrit dans une lettre adressée à Oliveira Martins que sa revue "a pour objectif de donner au Brésil une idée de ce qu'est le mouvement littéraire et artistique en Europe" ${ }^{20}$. Il recrute pour ce faire les "bons éléments dont le Portugal dispose", parmi lesquels figurent Monteiro Ramalho, Eça de Queirós ou Jaime de Séguier. D'abord rédigée et imprimée à Paris, puis à Lisbonne, la revue compte au total 184 numéros pour un tirage moyen de 15000 exemplaires, destinés principalement à un lectorat portugais. La réputation de son périodique doit beaucoup à la qualité des plumes engagées, des illustrations et de la mise en page, très soignée. La revue est également disponible par abonnement au siège de la Gazeta de Notícias à Rio de Janeiro, et ce journal carioca publie très régulièrement des annonces faisant la promotion de la publication ${ }^{21}$. Toutefois, du Brésil il n'est que peu question dans cette revue illustrée qui veut donner à voir à ses lecteurs portugais ou brésiliens ce que la «civilisation » en Europe a de meilleur.

Un exemple plus tardif, celui de Brasil-Portugal, revista quinzenal ilustrada (1899-1914), témoigne d'une ambition différente, celle de promouvoir cette confraternité lusobrésilienne dans ses colonnes :

L'idée est la suivante: faire connaitre le Brésil au Portugal, faire connaitre le Portugal au Brésil, en vulgarisant dans chacun de ces pays l'art et la littérature de l'autre, et en faisant apprécier de tous les écrivains et les artistes qui par le recours à une même langue, riche, sonore et rythmée, expriment le sentiment de la façon la plus intense et délicate et l'idée de la façon la plus profonde et brillante.

Castilho, Tavares \& Victor $1899: 2$

Dans cet article inaugural, les trois rédacteurs du Brasil-Portugal affirment également que leur ambition est de "maintenir et renforcer les relations de commerce et d'industrie entre les deux nations unies par le sang, par le sentiment et par la tradition". Soit une dimension plus pragmatique qui reflète l'ambivalence originelle d'une confraternité, d'une lusophonie essentialisée, certes, mais dotée d'objectifs pragmatiques sans lesquels il serait impossible de comprendre la genèse de cette "communauté imaginée" depuis Lisbonne. La revue lance une grande campagne de publicité au Brésil en 1900, à l'occasion du quatrième centenaire de la découverte du Brésil. Trois collaborateurs de la revue font le voyage au Brésil et participent aux commémorations officielles organisées à Rio de Janeiro. Un numéro exceptionnel du journal est tiré à cette occasion, offert gracieusement aux abonnés et vendu au public : sur 200 pages illustrées sont compilées une centaine d'articles inédits dont les auteurs sont à part égale portugais et brésiliens. Outre la revue bimensuelle, l'équipe de rédaction décide d'investir le marché alors très profitable de la presse populaire, à 
travers la publication de l'Almanaque do Brasil-Portugal, tiré en 1900 à 50000 exemplaires, dont on sait que 30000 étaient destinés au seul marché brésilien, via le réseau de correspondants dont dispose la revue dans les principales capitales d'État. La rédaction de cet almanach est le fruit elle aussi d'une étroite collaboration luso-brésilienne. En somme, voici l'exemple d'une revue dont le siège est à Lisbonne et dont les réseaux tissés de l'autre côté de l'Atlantique, notamment par Jaime Victor, alors également correspondant du Jornal do Brasil, permettent de pérenniser cette entreprise éditoriale là où beaucoup, avant cela, avaient périclité, faute de pouvoir assumer pleinement cette dimension luso-brésilienne susceptible de garantir un bon accueil au Brésil. Dans les colonnes du quotidien brésilien, Jaime Victor se satisfait du succès de la revue quatre années après sa fondation :

Il est juste de dire que le succès obtenu par cette entreprise littéraire et commerciale est due en grande partie au Brésil, non seulement aux fils de ce pays ami, mais aussi aux milliers de Portugais qui, éparpillés sur le vaste territoire de la République, ont toujours répondu gentiment et de manière patriotique aux appels qui leur ont été lancés par l'entreprise du Brasil-Portugal... ${ }^{22}$

\section{La colonie portugaise de Rio de Janeiro, relais de la lusitanité au Brésil}

$36 \mathrm{Au}$ sein de cette colonie portugaise de Rio de Janeiro, dont les effectifs croissent de manière remarquable dans la seconde moitié $\mathrm{du} \mathrm{XIX}^{\mathrm{e}}$ siècle, de nombreuses vocations littéraires vont également s'affirmer. En 1890, la colonie portugaise de Rio compte près de 100000 personnes, pour une population totale estimée à un demi-million d'habitants (Lessa 2002: 27). Bon nombre de ces immigrés sont employés dans le secteur du commerce portuaire, lequel est organisé en étroite dépendance avec les maisons de commerce du Portugal, et principalement de Porto (Alves 1994). Dans ces maisons de commerce travaillent de nombreux caixeiros, des commis de magasins dont l'intégration socio- professionnelle s'appuie sur des formes de solidarité parentale et intracommunautaire. En effet, la constitution de la "colonie portugaise" à Rio de Janeiro, principale communauté étrangère au Brésil au XIX ${ }^{\mathrm{e}}$ siècle, est contemporaine de la fondation de nombreuses associations culturelles, instructives, de bienfaisance ou, plus tard, mutualistes et sportives (Melo \& Silva 2009).

La présence portugaise dans le paysage culturel carioca ne se résume pas alors au fameux Real Gabinete português de Leitura, fondé à l'initiative de 43 commerçants portugais en 1837 afin d'instruire ses membres et promouvoir les sciences et les lettres portugaises au Brésil. Le Grêmio Literário Português no Rio de Janeiro est fondé en 1855, lorsque la revue littéraire $A$ Saudade se présente comme son organe de presse ${ }^{23}$. Cette association disparaît au milieu des années 1860, lorsque s'impose dans l'espace public un autre cercle, le Retiro Literário Português no Rio de Janeiro, fondé en 1859. À la différence du Gabinete Português de Leitura - dont l'histoire est, elle, bien connue -, ces nouvelles associations nourrissent des ambitions plus proprement littéraires, comme en témoigne leur politique éditoriale. Depuis la parution de la première série de la Saudade en 1855 jusqu'à la Revista do Retiro Literário Português, laquelle cesse de paraître en 1885, diverses publications périodiques ont permis à de jeunes lettrés venus du Portugal de se faire une place dans le paysage culturel carioca. Outre ces ambitions personnelles et collectives bien comprises, de telles revues ont également vocation à porter au-delà du cercle étroit des membres de leur société la voix de la culture 
portugaise au Brésil, alors que l'immigration portugaise croissante nourrit la résurgence de manifestations lusophobes dans l'Empire. Ici, le patriotisme portugais va de pair avec le souhait de porter haut le flambeau de l'esprit luso-brésilien à Rio de Janeiro. L'éloge fraternel des « progrès » du nouvel Empire est l'occasion de rappeler les vertus de l'héritage portugais laissé au Brésil ; une opinion en décalage avec le discours dominant de l'História Pátria produit au sein de l'Instituto Histórico e Geográfico Brasileiro (Guimarães 1988).

Outre l'édition de revues, ces deux associations littéraires recourent également au genre de l'album, une publication annuelle, afin de mettre en avant leurs talents littéraires respectifs tout en renouvelant l'appel à la confraternité. Dans l'Archivo do Retiro Literário Português no Rio de Janeiro (1870), le choix de l'alliance luso-brésilienne a pour pendant la condamnation explicite de l'ibérisme, alors en vogue chez certains intellectuels au Portugal. Deux auteurs dénoncent le leurre d'un rapprochement entre les deux monarchies ibériques, une union qu'ils analysent comme la reddition annoncée du Portugal à son ennemi de toujours (Muniz 1870, Gouvêa 1870).

C'est en outre à un membre du Grêmio Literário Português, Faustino Xavier de Novais, qu'il revient de fonder une revue qui aspire à exalter la fraternité luso-brésilienne. Si elle n'est pas dépendante d'une association littéraire, la revue $O$ Futuro (1862-1863) bénéficie au cours de sa brève existence des relations privilégiées tissées par son directeur au sein du Grêmio et, aussi, dans le petit monde des lettres carioca. Novais y publie de nombreux auteurs des romantismes portugais et brésilien, parmi lesquels José de Alencar, Quintino Bocaiúva, Joaquim Manuel de Macedo, Henrique César Muzzio ou Francisco Otaviano, tout en ouvrant les colonnes de sa revue à ces lettrés portugais installés à Rio de Janeiro, comme Reinaldo Carlos Montoro ou Augusto Emílio Zaluar.

L'intégration de ces associations portugaises dans le paysage culturel carioca passe également par la célébration de fêtes commémoratives. En 1862, le Retiro organise une grande soirée littéraire et artistique en présence de 500 personnes, sous la présidence du consul général du Portugal. Ce sarau, dont le déroulé est ponctué de nombreux discours, poésies et intermèdes musicaux, s'achève, nous précise-t-on, à $4 \mathrm{~h}$ du matin. Le chroniqueur du Diário do Rio de Janeiro ne manque pas de saluer les hommes de lettres portugais qui ont apporté leur concours, comme Augusto Emílio Zaluar, Faustino Xavier de Novais ou Reinaldo Carlos Montoro, alors directeur du Grêmio Literário português ${ }^{24}$. Un tel succès tient, pour partie, à la réputation littéraire et à la réussite sociale de quelques-uns des jeunes fondateurs du Retiro, dont le succès personnel se prolonge sur le plan symbolique par la tenue de tels événements fastueux qui honorent le cercle littéraire et la colonie portugaise en son ensemble - comme l'atteste la présence de ses représentants les plus éminents. Les événements auxquels le Retiro s'associe au cours de cette décennie exaltent les valeurs de la lusitanité. Qu'il s'agisse de souscrire à l'élévation d'un monument en l'honneur de Camões en $1860^{25}$ ou d'organiser des festivités en l'honneur du mariage de D. Luiz I avec la princesse de Savoie en $1862^{26}$, les occasions ne manquent pas de rappeler l'engagement du Retiro au service de la monarchie portugaise et de la gloire de ses lettres. Ces associations assument donc une politique de promotion des intérêts portugais à Rio de Janeiro dont l'efficacité est conditionnée à l'exaltation des liens confraternels sur lesquels se fonde le postulat d'une relation privilégiée entre le Portugal et le Brésil ${ }^{27}$.

41 L'année 1880 est un marqueur important dans cette archéologie d'une communauté littéraire luso-brésilienne. À Rio de Janeiro, plusieurs célébrations sont organisées à 
l'initiative conjointe des hommes de lettres cariocas, du pouvoir impérial et de la communauté portugaise (Descrição 1880). Les associations portugaises jouent un rôle de premier plan dans l'organisation des festivités du Tricentenaire de la mort de Camões, dont l'exaltation de la mémoire de part et d'autre de l'Atlantique vient vivifier ce patrimoine commun, luso-brésilien. De telles mondanités suscitent des échos enthousiastes au Portugal où, certes, les gouvernements successifs s'inquiètent de cette hémorragie démographique que constitue l'émigration à destination du Brésil, mais où l'orgueil national se flatte de la réussite sociale et du patriotisme des membres des associations portugaises d'outre-mer. D'ailleurs, le Retiro Literário Português reste actif jusqu'aux premières années $\mathrm{du} \mathrm{xx}^{\mathrm{e}}$ siècle comme il profite aussi de l'essor d'une presse portugaise dont le nombre de titres croît de façon tout à fait remarquable dans le Rio de Janeiro fin-de-siècle ${ }^{28}$. C'est donc bien cette diversification des formes de publicité et de socialisation qui rend la politique éditoriale initiée dans les années 1850 caduque: l'essor de la presse portugaise et l'écho croissant dont bénéficient les principales associations portugaises dans l'espace public carioca suffisent à assurer la promotion des activités et événements auxquels le Retiro associe son nom. Ce faisant, cette société pérennise des relations cordiales avec des institutions et associations culturelles brésiliennes, au nom d'une confraternité luso-brésilienne bien réelle : la réciprocité sur le long terme des échanges et des invitations mondaines marque la vigueur de ces liens entre sociétés portugaises et brésiliennes à Rio de Janeiro, au-delà des postures nationalistes affichées de manière récurrente par ces mêmes acteurs.

\section{Panlusisme, portugalité ou lusophonie avant la lettre : le choix des mots}

Le constat semble assez clair. C'est d'abord parce que le Brésil a été perçu comme une terre d'avenir pour ces écrivains portugais soucieux de leur carrière littéraire que l'on voit émerger dans les imaginaires transatlantiques cette communauté qui n'est pas une nation, qui ne peut plus être un Empire, mais qui incarnerait une nouvelle modalité de la coexistence au sein de ce "lusotope" nouveau que constituent au xix siècle le Portugal et le Brésil réunis. Un tel imaginaire lusophone a eu des échos au Brésil, bien sûr : qu'il s'agisse de souligner le rôle prééminent des lettrés de la colonie portugaise ou que l'on s'appesantisse sur la circulation remarquable de la littérature portugaise au Brésil, de nombreux signes tangibles témoignent des affinités électives qui unissent ces deux pays, au-delà des incompréhensions et des rivalités récurrentes.

Pour ces écrivains portugais, il s'est moins agi de gagner le Brésil, où les conditions de vie des hommes de lettres n'étaient guère plus enviables, que de mettre en œuvre une stratégie cumulative des tâches et des revenus, en profitant du partage en commun d'une langue, d'un passé historique et d'une curiosité réelle au Brésil pour la création littéraire portugaise. Rappelons d'ailleurs que la relation luso-brésilienne n'épuise jamais l'éventail des relations culturelles internationales dans lesquelles agissent ces acteurs. Déjà, cette lusophonie avant la lettre est un "espace spécifique d'intersection avec d'autres identités", comme le rappelle Eduardo Lourenço ${ }^{29}$, dont les frontières, largement virtuelles, ne sont pas étanches, surtout en un siècle où la France reste un référent de poids. Il n'en reste pas moins que l'essor des importations de livres en provenance du Portugal, la présence remarquée des écrivains portugais dans la presse du Brésil ou les dénonciations récurrentes des abus de la contrefaçon, témoignent de 
l'intérêt croissant des hommes de lettres et du livre portugais pour le marché brésilien. Par ailleurs, il ne fait aucun doute que l'essor de l'émigration portugaise à destination du Brésil a constitué un marché de niche pour ces écrivains portugais qui trouvaient là des lecteurs nombreux. La presse luso-brésilienne, à Lisbonne, s'est développée à partir d'un postulat identique. Cet investissement premier et récurrent des Portugais tend donc à faire de cette communauté lusophone avant la lettre un prolongement postimpérial du Portugal hors de ses modestes frontières ibériques, la manifestation parfois trop clairement énoncée d'une "portugalité" (Cahen 2008) qui a suscité au Brésil des réactions parfois bienveillantes, souvent indifférentes voire carrément hostiles.

Cette communauté lusophone est donc mobilisée dans les discours et les pratiques par des hommes de lettres, des publicistes et des libraires-éditeurs portugais et, dans une moindre mesure, brésiliens. Ces acteurs constituent une élite culturelle lettrée appartenant aux classes supérieures, moyennes et, plus rarement, populaires, des sociétés portugaise et brésilienne ${ }^{30}$. Dans leur très large majorité, soulignons-le aussi, ces hommes de lettres sont d'ascendance européenne : vu du Brésil, le lien privilégié avec le Portugal permettait aux élites impériales d'ascendance européenne de cultiver cette filiation assumée et revendiquée entre le Brésil indépendant et le Vieux Continent. Ce qui n'interdit pas que des écrivains brésiliens d'origine métisse ou pardos aient joué le cas échéant un rôle important dans cette communauté luso-brésilienne, tels Gonçalves Dias, Gonçalves Crespo ou Machado de Assis.

La vitalité de cet espace luso-brésilien ne peut s'expliquer que par le seul activisme de quelques dizaines de lettrés et hommes du livre de part et d'autre de l'Atlantique. Cet espace n'aurait pu exister sans l'essor concomitant d'un public, d'un lectorat ouvert aux créations littéraires en langue portugaise, qu'elles soient d'origine portugaise ou brésilienne. Il ne faut pas mésestimer ainsi la grande audience dont ont bénéficié les essayistes, les romanciers, les dramaturges et les poètes portugais au Brésil ${ }^{31}$. Leurs romans, leurs chroniques dans la presse, leurs créations dramatiques, leurs compositions poétiques ont circulé comme jamais auparavant au Brésil, où le lectorat connaît une croissance suffisamment remarquable pour susciter les convoitises des écrivains portugais, comme nous l'avons vu. Et si cette lusophonie avant la lettre pénètre plus difficilement les couches populaires, métisses et esclaves du Brésil, il ne faudrait pas oublier l'importance que revêtent à cette époque la lecture à voix haute et la mise en musique de la poésie dans la diffusion vers le plus grand nombre des œuvres littéraires au sein de cette société brésilienne si profondément imprégnée par l'analphabétisme et l'esclavage.

Cette communauté est née dans les imaginaires comme elle s'est aussi traduite dans les faits, pour défendre les intérêts de ses membres, qu'ils soient individuels, nationaux voire, aussi, internationaux. Cette communauté ouverte, à laquelle, faut-il le rappeler, nombre d'hommes de lettres n'ont pas adhéré, a agrégé à elle les bonnes volontés intéressées à l'essor de ces relations privilégiées bilatérales à une époque où la culture est un puissant vecteur de la mondialisation (Charle 2015). Elle est donc d'abord le résultat du croisement de logiques interpersonnelles, dans lesquelles l'État n'a pas joué un rôle moteur, à une époque où la diplomatie culturelle de ces deux pays en est encore à ses balbutiements et ne dispose ni d'une base théorique clairement établie ni des moyens matériels susceptibles de promouvoir comme certains aspirent pourtant déjà à le faire la voix des littératures de langue portugaise dans l'espace littéraire international ${ }^{32}$. 

brésiliens trouve son prolongement au siècle suivant, tout en prenant des configurations nouvelles, puisque cette relation privilégiée n'a jamais été exclusive : le Brésil tend à se détourner de plus en plus de l'Europe au sortir de la Grande Guerre (Compagnon 2013) ; la Semaine d'art moderne, à São Paulo en 1922, est l'occasion pour une nouvelle génération d'artistes et d'écrivains de refonder sur des bases nouvelles la culture et de l'identité brésiliennes, au prix d'une distanciation plus nette vis-à-vis des modèles européens; quand, cette même année, d'autres cultivent à l'occasion des commémorations du centenaire de l'indépendance et de la visite du Président de la République portugaise la fraternité luso-brésilienne. Quant au Portugal, si la proclamation de la République en 1910 est l'occasion d'un rapprochement diplomatique avec le Brésil, qui mobilise notamment un discours «panlusitaniste» (Ferreira 2010: 84), l'avènement au pouvoir de Salazar marque à la fois un net repli du Portugal sur luimême et le renouveau de l'idéologie impérialiste en Afrique, sans que la relation avec le Brésil soit jamais tout à fait négligée. Celle-ci se fait à la fois plus réelle et prosaïque, et la communauté de destin semble se déliter peu à peu, tant la société brésilienne semble se distancier de plus en plus du Portugal sur le plan culturel voire symbolique, en dépit de la pérennisation des voies de l'émigration portugaise vers le Brésil.

\section{BIBLIOGRAPHIE}

\section{Sources et bibliographie}

Almeida, I. d' 1879, “O movimento litterario recente em Portugal”, União academica, 11 : 5.

Alves, J. F. 1994, Os brasileiros, emigração e retorno no porto Oitocentista, Porto, Gráficos reunidos.

Berrini, B. 2003, Brasil e Portugal : a geração de 70, Porto, Campo das Letras.

Blake, A. V. Alves do Sacramento 1883-1902, Dicionário bibliográphico brazileiro, Rio de Janeiro, Imprensa nacional, 7 vols.

Bonilha, J. 2015, Eça de Queirós e a Gazeta de Notícias : Supplemento literário 1892, Jundiaí, Paco Editorial.

Branco, C. Castelo 1879, Os Criticos do "Cancioneiro alegre", Porto, E. Chardron.

Cahen, M. 1997, “Des caravelles pour le futur? Discours politique et idéologie dans

l' 'institutionnalisation' de la Communauté des pays de langue portugaise", Lusotopie, 4 : 391-431.

Cahen, M. 2008, "Persistance du lusotropicalisme au XXI" siècle", Lusotopie, XV (2) : 223-229.

Casanova, P. 2008, La République mondiale des lettres, Paris, Seuil.

Castilho, A. de, Tavares, Lorjo \& Victor, J. 1899, “A nossa apresentação”, Brasil-Portugal, $1: 2$.

Chagas, M. J. Pinheiro 1879, A Propriedade litteraria. Carta a sua magestade o imperador do Brazil, Porto, Livraria internacional de Ernesto Chardron : 63-69. 
Charle, C. 2015, La dérégulation culturelle. Essai d'histoire des cultures en Europe au XIXe siècle, Paris, PUF.

Coelho, J. M. Vaz Pinto 1880, “Da propriedade litteraria no Brazil”, Revista brazileira, 6.

Compagnon, O. 2013, L'Adieu à l'Europe. L'Amérique latine et la Grande Guerre (Argentine et Brésil, 1914-1939), Paris, Fayard.

Descrição da festa comemorativa do tricentenario de Camões 1880, Rio de Janeiro, Tipographia de J. D. de Oliveira.

Dicionário (0) bibliográphico português julgado pela imprensa contemporanea nacional e estrangeira, Lisboa, Imprensa nacional, 1867, p. 134.

Ferreira, M.-J. 2010, “Portugal-Brésil (1910-1922) : refonder une politique d'espace de voisinage culturel”, Matériaux pour l'histoire de notre temps, I (97-98) : 82-90.

Gouvêa, F. de P. Santos 1870, “Brado patriótico”, Archivo do Retiro Litterario Portuguez : 177-179.

Guimarães, M. L. Salgado 1988, “Nação e civilização nos trópicos: o Instituto Histórico e Geográfico Brasileiro e o projeto de uma história nacional”, Estudos Históricos, 1 : 5-27.

Kalifa, D. et alii eds. 2011, La civilisation du journal. Histoire culturelle et littéraire de la presse française au XIX ${ }^{e}$ siècle, Paris, Nouveau Monde Éditions.

Leal, C. E. 2015, “Gazeta de Notícias”, in A. Alves de Abreu ed., Dicionário histórico-biográfico da Primeira República (1889-1930), Rio de Janeiro, Editora CPDOC.

Lessa, C. 2002, “Rio, uma cidade portuguesa?”, in C. Lessa ed., Os Lusíadas na aventura do Rio moderno, Rio de Janeiro, Record/Faperj.

Lourenço, E. 1999, A Nau de Ícaro seguido de imagem e miragem da lusofonia, Lisboa, Gradiva.

Luca, T. R. de 2014, “A Illustração (1884-1892) : algumas questões teórico-metodológicas” in M. Abreu \& M. Midori Deaecto eds., A Circulação transatlântica dos impressos : conexões, Campinas/São Paulo, Unicamp/IEL : 167-174.

Melo, D. \& Silva, E. C. da eds. 2009, Construção da nação e associativismo na emigração portuguesa, Lisboa, Imprensa de Ciências Sociais.

Mendes, J. Sacchetta Ramos 2010, Laços de sangue. Privilégios e intolerância à imigração portuguesa no Brasil (1822-1945), Porto, Fronteira do Caos e CEPESE.

Muniz, P. 1870, “A União ibérica”, Archivo do Retiro Litterario Portuguez do Rio de Janeiro : 32-67.

Pereira, V. \& Carlos, J. 2006, “Les relations culturelles luso-brésiliennes et la conquête du marché du livre par les auteurs portugais et brésiliens au temps de Camilo Castelo Branco", in C. Poncioni \& J. M. Esteves eds., Au Carrefour des Littératures Brésilienne et Portugaise : influences, correspondances, échanges (XIXe et XXe siècles), Paris, Lusophone : 351-374.

Rozeaux, S. 2014a, "Splendeurs et misères du 'siècle de dom Pedro II' : le mécénat impérial et les Letras Pátrias au Brésil (1840-1889)”, Romantisme, 2 (164) : 107-119.

Rozeaux, S. 2014b, "Un patriotisme brésilien à géométrie variable? La renaissance des 'petites patries' au prisme des Letras Pátrias (1850-1880)”, Brésil(s), 6 : 181-201.

Rozeaux, S. 2015a, “Devenir correspondant de la presse brésilienne en Europe : Anatomie sociale et circulations d'une nouvelle figure du paysage médiatique dans l'espace atlantique (fin XIX ${ }^{\mathrm{e}}$ début $\mathrm{XX}^{\mathrm{e}}$ siècles)", in G. Pinson et M.-E. Thérenty eds., La presse et les journalistes au XIX ${ }^{e}$ siècle : 
identités et modernités, Paris, Médias19, consulté le 24 mai 2018, http://www.medias19.org/ index.php?id=23162.

Rozeaux, S. 2015b, "Les fondations fragiles d'un espace littéraire transatlantique : les lettres brésiliennes dans le Dicionário bibliográfico português d'Inocêncio Francisco da Silva (1858-1883)”, Cultura. Revista de história e teoria das ideias, 34 : 149-174.

Rozeaux, S. 2015c, "Réceptions croisées du Cancioneiro alegre de poetas portuguezes e brazileiros (1879) de Camilo Castelo Branco : la formation d'un espace littéraire luso-brésilien sous tension", Amnis. Revue de civilisation contemporaine Europes/ Amériques, 14, consulté le 24 mai 2018, http:// journals.openedition.org/amnis/2550 et DOI : 10.4000/amnis.2550.

Rozeaux, S. 2016, "Presença da colónia portuguesa na paisagem cultural e mediática do Rio de Janeiro : o Grêmio Literário Português e o Retiro Literário Português (1855-1885)", Topoi, 17 (33) : 490-513.

Rozeaux, S. 2017, "La mobilisation transatlantique des hommes de lettres en faveur de la reconnaissance du droit d'auteur au Brésil (1851-1898)", Le Mouvement social. Revue d'histoire sociale, 1 (258) : 49-66.

Silva, I. F. da 1858-1870, Diccionario bibliographico portuguez. Estudos de Innocencio Francisco da Silva applicaveis a Portugal e ao Brasil, Lisboa, Imprensa nacional, 9 vols.

Silva, M. B. Nizza da 1992, Documentos para a História da Imigração Portuguesa no Brasil, 1850-1938, Rio de Janeiro, Federação das Associações Portuguesas e Luso-Brasileiras.

Wolf, F. 1863, Le Brésil littéraire. Histoire de la littérature brésilienne suivie d'un choix de morceaux tirés des meilleurs auteurs brésiliens, Berlin, A. Ascher \& Co.

Zan, J. C. 2009, Ramalho Ortigão e o Brasil, Thèse de lettres, Université de São Paulo.

\section{Journaux}

Cidade do Rio

Correio Mercantil

Diario do Rio de Janeiro

Farpas (As). Chronica mensal da politica, das letras e dos costumes

Gazeta de Notícias

Jornal do Brasil

Revista Brazileira

Revista do Instituto Histórico e Geográfico Brasileiro

Revista illustrada

Revista popular

Saudade (A). Publicação litteraria e instructiva

\section{NOTES}

1. Nous n'entrerons pas ici dans le débat historiographique consistant à interroger la nature de ce processus d'indépendance au Brésil et le fait de savoir s'il est justifié de parler dans ce cas 
précis de décolonisation, compte tenu du fait que les structures économiques et sociales en place au XIX ${ }^{\mathrm{e}}$ siècle sont largement héritées de la période coloniale. Nous utilisons donc le qualificatif de "post-colonial" pour signifier ici d'abord la rupture politique avec le Portugal en 1822.

2. L'expression consacrée de Letras Pátrias, dont l'usage se répand parmi les hommes de lettres, traduit alors le caractère éminemment national de la littérature produite par les membres de ce courant romantique.

3. Revista do Instituto Histórico e Geográfico Brasileiro, 1859, vol. XXII : 72.

4. Revista popular, 15 septembre 1860, no $7: 357$.

5. Voir, pour de plus amples considérations Rozeaux (2015b).

6. Voir, à ce sujet Pereira \& Carlos (2006).

7. Revista illustrada, Rio de Janeiro, $\mathrm{n}^{\mathrm{o}} 6,1879: 7$.

8. Ibid., p. 50.

9. As Farpas. Chronica mensal da politica, das letras e dos costumes, Lisbonne, Typographia Universal, 1871-1883.

10. Revista Brazileira, vol. $1,1879: 281$.

11. Pour de plus amples considérations, voir Rozeaux (2015c).

12. Correio Mercantil du 4 août 1866.

13. Ce recensement fait état d'un taux d'alphabétisation de $18 \%$ parmi la population libre du pays. Cependant, ce taux atteint près de $50 \%$ à Rio de Janeiro, et on observe des taux élevés dans les principales capitales de province.

14. As Farpas, juillet 1872, p. 10.

15. As Farpas, décembre 1872, p. 13.

16. Cidade do Rio, 22 octobre $1887, \mathrm{n}^{\mathrm{o}} 25: 1$.

17. Gazeta de Notícias, 24 juillet $1880: 1$.

18. Rappelons que ce dernier était alors ambassadeur du Portugal à Paris, un poste qu'il occupait depuis 1888 et ce, jusqu'à son décès dans sa maison de Neuilly-sur-Seine en 1900.

19. Gazeta de Notícias, 25 août 1894, no $236: 1$.

20. Lettre reproduite dans Berrini (2003 : 111). Lettre de Mariano Pina du 3 mars 1885 adressée depuis Paris à Oliveira Martins.

21. Sur les relations complexes entre ces deux titres de la presse, voir Luca (2014).

22. Jornal do Brasil, 16 mars $1902: 2$.

23. A Saudade: publicação litteraria e instructiva, Rio de Janeiro, Typographia de F. A. de Almeida, 1855-1857.

24. Diario do Rio de Janeiro, 21 de Julho de $1862: 1$.

25. Correio Mercantil, 5 décembre $1860: 2$.

26. Te-Deum. Correio Mercantil, 7 novembre $1862: 2$.

27. Sur ces associations littéraires portugaises de Rio de Janeiro, voir également Rozeaux (2016).

28. Pour un relevé des titres de la presse portugaise du Brésil, voir Silva (1992 : 114-115).

29. Voir, en particulier Lourenço (1999).

30. Rappelons ici que les membres de la colonie portugaise de Rio de Janeiro étaient dans leur majorité issus des couches les plus modestes de la société portugaise. En outre, nombre d'hommes de lettres brésiliens au XIX ${ }^{\mathrm{e}}$ siècle appartiennent aux classes moyennes et populaires urbaines, à l'instar de Gonçalves Dias, Joaquim Manuel de Macedo ou Machado de Assis. Les écrivains issus de l'aristocratie et de grandes familles de propriétaires terriens, tels José de Alencar ou Joaquim Nabuco, constituent en réalité une minorité.

31. La réciproque n'est pas aussi évidente, même si les œuvres de Gonçalves Dias, par exemple, ont reçu un très bon accueil au Portugal.

32. Sur la mise en place de cette diplomatie culturelle entre le Brésil et le Portugal, à l'initia-tive de ce dernier, voir Ferreira (2010). 


\section{RÉSUMÉS}

L'indépendance du Brésil en 1822 reconfigure les relations et circulations culturelles entre le Portugal et son ancienne colonie. À compter du milieu du siècle, ces relations se font plus intenses, alors que l'émigration portugaise à destination du Brésil ne cesse de croître. Ce payscontinent attire les migrants comme il suscite les convoitises des hommes de lettres portugais qui perçoivent le Brésil comme une "terre d'avenir", une aubaine pour leur carrière, compte tenu des liens confraternels qu'ils cultivent et entretiennent avec leurs compères et le lectorat brésiliens, au nom d'une culture, d'une langue ou d'une histoire qu'ils auraient en partage.

A independência do Brasil em 1822 reconfigura as relações e circulações culturais entre Portugal e sua antiga colônia. Desde meados do século XIX, essas relações se intensificam, à medida que a emigração portuguesa com destino ao Brasil não para de crecer. Este país continente atrai os migrantes, bem como suscita a cobiça dos homens de letras portugueses que enxergam o Brasil como um "país do futuro", uma nova oportunidade para as suas carreiras, considerando os laços confraternais que eles cultivam e mantém com seus parceiros e com os leitores brasileiros em nome de uma cultura, de uma língua ou de uma história supostamente comum.

Brazil's Independence in 1822 reshaped the cultural relationships and circulations between Portugal and its former colony. From the mid- $19^{\text {th }}$ century on, these relationships become more intense, while the Portuguese emigration to Brazil continues to grow. This country, huge as a continent, welcomes migrants as well as arouses the desires of the Portuguese men of letters who perceive Brazil as a "land of the future", an opportunity to their career, given the peer bonds they cultivate and maintain with their Brazilian colleagues and readers, in the name of a culture, a language or a story they would share.

\section{INDEX}

Palavras-chave : edição, homens de letras, imprensa, lusofonia, século XIX

Keywords : 19 th century, lusophony, men of letters, newspapers, publishing

Mots-clés : édition, hommes de lettres, lusophonie, presse, XIXe siècle

\section{AUTEUR}

\section{SÉBASTIEN ROZEAUX}

Maître de conférence en histoire, FRAMESPA, Université Toulouse Jean Jaurès, France sebastien.rozeaux[at]univ-tlse2.fr 